\title{
Fibromyalgia and vibratory platform exercise: Cortisol improvement and women's quality of life
}

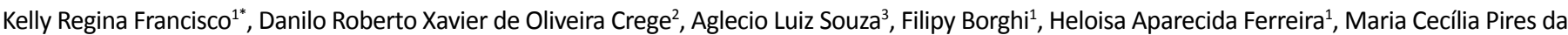 \\ Rocha $^{1}$, Hidetake Imasato ${ }^{4}$, Priscila Cristina da Silva ${ }^{1}$ and Dora Maria Grassi-Kassisse ${ }^{1}$
}

\begin{abstract}
Francisco KR, Crege DRXO, Souza AL, et al. Fibromyalgia and vibratory platform exercise: Cortisol improvement and women's quality of life. Curr Res Integr Med 2017;2(2):17-20.
\end{abstract}

OBJECTIVES: The main objective of this pilot study was to evaluate the effects of an exercise protocol, using vibratory platform, in quality of life and the cortisol rhythmicity in fibromyalgic women.

METHODS: Twenty women, which 10 were healthy and 10 were diagnosed with fibromyalgia. All of them performed an exercise program on a vibratory platform and were evaluated for salivary cortisol production throughout the day before and after the treatment. The volunteers with fibromyalgia were also evaluated with a specific questionnaire for quality of life before and after the treatment. Salivary samples were collected at 6 a.m., noon, 6 p.m. and before bedtime, 10 p.m. Cortisol was quantified using immunoenzymatic assay.

RESULTS: Women with fibromyalgia improved their quality of life and started to have an adjustment in the cortisol rhythmicity after exercise. Even considering the limitations of the volunteers' number who participated in this pilot, a relationship between the improvement of the quality of life and the adjustment in the cortisol rhythmicity produced by the exercise program practiced could be assumed.

CONCLUSION: The results suggest that the proposed training program can be considered as a further resource as an adjunct in the treatment of the fibromyalgic population, thus contributing to the eustress, including in the healthy volunteers group.

Key Words: Cortisol, Fibromyalgia, Quality of life, Vibratory platform
Cortisol acts physiologically in the body to keep it in balance with the day by day challenges. This hormone is essential for eustress (healthy stress) and can be damaging to the body in distress (stress that causes diseases). Eustress is the daily motivation that encourages us to face new challenges $(1,2)$. The activation of the endocrine pathway is so important for the proper functioning of vital functions that, if animals do not have this regulation system, any normally harmless stressor agent would lead to death. This phenomenon has been proven in studies with adrenalectomized animals (3).

As described by Selye (4), the reaction to chronic stress develops the general adaptation syndrome, a theory that describes the body's response to any stressor. This response is divided into three phases: alarm, resistance and exhaustion. The alarm phase is one that occurs as soon as the individual comes in contact with the possible threat, triggering the activation of the sympathetic nervous system and the activity of the adrenal gland. With the maintenance of the stressor, the response of the organism enters in the resistance phase, where the return to equilibrium is sought through adaptive mechanisms. Finally, if this adaptation is not sufficient for the rebalancing or the stressor remains, the individual enters in the third stage, the exhaustion, where the development of diseases occurs, the distress $(2,5-8)$.

There are several diseases related to chronic stress and, therefore, to the inadequate functioning of the hypothalamic-pituitary-adrenal axis. Fibromyalgia is one of them, a potentially disabling syndrome whose prevalence reaches values between 0.66 and $4.4 \%$ and affects eight times more women than men (9). This disturb has a still undefined etiology and its treatment is based on the control of symptoms and physiological changes. The main symptoms include muscle pain, sleep disorders, fatigue and stress, very similar conditions to those observed in individuals who exhibit a misalignment between the circadian rhythm and the sleep wake cycle (10).

The proper cortisol release cycle occurs decreasingly during the day, with a peak about 30 minutes after waking in the morning and its low at around midnight (1). According to Riva et al. (11), patients with fibromyalgia present this altered cycle, with lower concentrations of cortisol in the morning and little variation throughout the day.

Regarding the treatment of the syndrome, some low-impact and low-load exercises have been shown to be effective and do not produce side effects like some medications, and significantly reduce symptoms (12-14).

Considering the possible correlation between the altered cortisol release, the pathophysiology of the disease and the beneficial effect of exercises, the aim of this study was to evaluate the rhythmicity of cortisol and the quality of life of women with fibromyalgia before and after a program of exercises in vibratory platform.

\section{METHODS}

The pilot has the participation of 20 women, which 10 were healthy and 10 were diagnosed with fibromyalgia. All the volunteers signed the "Free and Informed Consent Term" (CAAE: 0155.0.135.000-10). Women with thyroid dysfunction, hypertension, diabetes mellitus, renal dysfunction, hepatic function or anemia were not included in this study.

\section{Exercise program}

Both groups performed an exercise protocol on the vibratory platform (60 $\mathrm{Hz}$, acceleration $0.6 \mathrm{~g}$ and amplitude $0.4 \mathrm{~mm}$ ) two sessions per week, for a period of eight weeks. Each exercise lasted for 30 seconds and was repeated 6 times, 3 minutes between each activity for recovery. At the first 2 sessions, the groups performed only the first 3 exercises for 3 times, for adaptation. The protocol followed was described by Geli et al. (15). (a) static squat at $100^{\circ}$ of knee flexion; (b) dynamic squat between $90^{\circ}$ and $130^{\circ}$ of knee flexion; (c) maintained ankle plantar-flexion with legs in extension; (d) flexo-extension of the right leg between $100^{\circ}$ and $130^{\circ}$ of knee flexion; (e) flexo-extension of the left leg between $100^{\circ}$ and $130^{\circ}$ of knee flexion; and (f) squat at $100^{\circ}$ of knee flexion shifting the body weight from 1 leg to the other.

\section{Assessments}

\section{A) Fibromyalgia Impact Questionnaire - FIQ}

The FIQ is an evaluation instrument used in clinical practice and in scientific research to quantify the quality of life of individuals with fibromyalgia. This is a questionnaire with 10 items that assess the functional capacity, the work status, psychological disorders and physical symptoms. The score ranges from 0 to 100 and higher scores indicate greater impact of fibromyalgia on functioning. Only the volunteers with fibromyalgia were evaluated in relation to this variable (16).

\section{B) Salivary cortisol concentration analysis}

Participants were instructed to collect saliva samples at home, whose sallivete were to be stored under refrigeration. The cortisol rhythmicity was evaluated

${ }^{1}$ Laboratory of Stress Study,Structural and Functional Biology Department, Biology Institute, University of Campinas, Campinas, Brazil, 2Padre Anchieta University Center, Jundiai,Brazil, 3Metabolic Unit, Faculty of Medical Sciences, University of Campinas, Campinas, Brazil, 4Instituto de Quimica de São Carlos Universidade de São Paulo, São Carlos, Brazil

Correspondence: Dr. Kelly Regina Francisco, Laboratory of Stress Study (LABEEST), Department of Structural and Functional Biology, Biology Institute, State University of Campinas (UNICAMP), CP 6109, Cidade Universitária Zeferino Vaz, Rua Monteiro Lobato, 255 - Campinas, São Paulo, Brasil - CEP 13083-862, Telephone +55. 19-3521-6186,E-mail kellysaocarlos@hotmail.com

Received: June 20, 2017, Accepted: July 26, 2017, Published: August 03, 2017 
by saliva collections at four times of the day, upon awakening ( 6 a.m.), before lunch (12 a.m.), before dinner (6 p.m.) and before bedtime (10 p.m.). On the day after collection, the samples were centrifuged at $2800 \mathrm{rpm}$, at $4^{\circ} \mathrm{C}$ and for 20 minutes and analyzed using the commercial ELISA kit (Diagnostics Biochem Canada Inc. - Ref CAN-C-290) at the Laboratory of Stress Studies (LABEEST), at UNICAMP, as conducted by Rocha et al. (17). The results were expressed in $\mathrm{ng} / \mathrm{mL}$ for each sample and in area under the curve for the four saliva samples.

\section{C) Statistical analysis}

After normality test performed by D'Agostino \& Person, the data that presented normality were analyzed by Anova followed by Tukey or paired or unpaired Student $t$ test. The data that did not show normality were analyzed by Kruskall-Wallis followed by Dunn's, Wilcoxon or MannWhitney. Correlation analysis was performed by Pearson. Statistical analyzes and figures were performed using GraphPad Prism software version 5.00 for Windows (GraphPad Software, San Diego California USA). Data were considered significantly different when $p$ values were less or equal than 0.05 .

\section{RESULTS}

The FIQ values were significantly reduced after the exercise program, suggesting that this program promoted improvements in the quality of life of fibromyalgic women $(\mathrm{p}=0.0014$, Figure 1$)$.

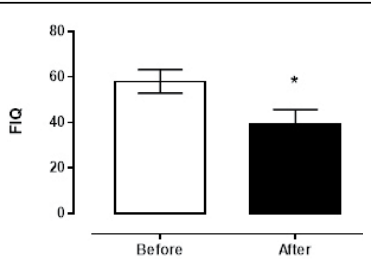

Figure 1) Fibromyalgia Impact Questionnaire (FIQ) scores before and after the vibratory platform exercise program. Ten volunteers participated in the exercise program over eight weeks. The results presented normality and were analyzed by the Student $t$ test, $p=0.0014$.

The volunteers in the control group (Figure 2A) showed rhythmicity of cortisol production before the beginning of the exercise program, and cortisol values collected at 6:00 p.m. and 10:00 p.m. were significantly lower than those produced upon awakening. After the exercise program, the control women had maintained cortisol rhythmicity; however, there was a significant increase in the cortisol values produced in the morning (6h, Figure 2A).

Volunteers with fibromyalgia prior to the exercise program presented altered rhythmicity in the cortisol production, since only the $10 \mathrm{p} . \mathrm{m}$. sample was significantly lower than the one produced at upon awakening. After the exercise program, the fibromyalgic volunteers showed an adjustment in the cortisol rhythm, showing a significant decrease in cortisol production at 6:00 p.m., compared to the values collected upon awakening at 6:00 a.m. (Figure 2B).
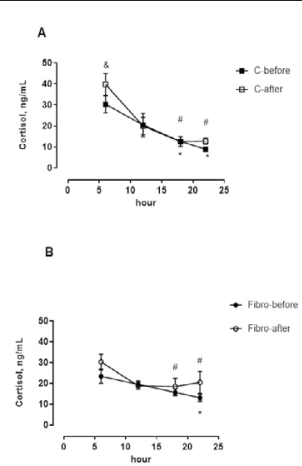

Figure 2) Rhythmicity of cortisol production in healthy women (C) and women with fibromyalgia (Fibro) before (before) and after an exercise program in vibratory platform. Cortisol concentration $(\mathrm{ng} / \mathrm{mL})$ was quantified in saliva samples collected at four different times throughout the day: upon waking (6 a.m.), before lunch (12 a.m.), before dinner (6 p.m.), and before bedtime (10 p.m.) before and after the exercise program. Data from the volunteers control (before/after) and fibro (before) were analyzed by Kruskall-Wallis followed by Dunn's being * $p<0.05$ versus 6 a.m. day before; And \# $p<0.056$ p.m. and 10 p. m. vs. 6 a.m. later. The data of the fibro volunteers (after) were analyzed by Anova followed by Tukey being \# $p<0.056$ a.m. and 10 p.m. vs. 6 a.m. later. The waking values were evaluated by the Student $t$ test, $\mathcal{E} p<0.05$.
Cortisol was also evaluated by area under the curve (AUC, ng/mL). The significant alteration in cortisol production at different times does not interfere in the total amount of cortisol produced between populations or after exercise (Figure 3).

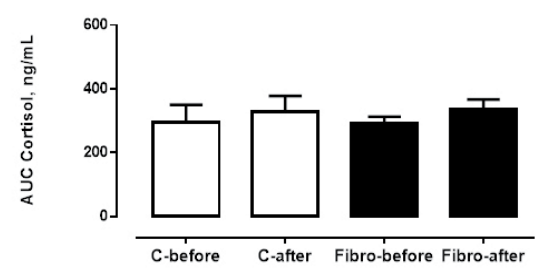

Figure 3) Daily amount of cortisol (AUC, $n g / m L$ ) produced in healthy (C) and fibromyalgic (Fibro) women before and after the vibratory platform exercise program. The AUC was calculated from the salivary cortisol concentration analyzed in four saliva samples obtained on two separate days, before and after the exercise program. The collection times were: upon awakening (6 a.m.), before lunch (12 a.m.), before dinner (6 p.m.) and before bedtime (10 p.m.). Statistical analyzes after vs. before, Wilcoxon for control and Student's $t$ test for fibro, before ws. before and after vs. after the test used was Mann-Whitney

In order to evaluate a possible correlation between improvement in quality of life and cortisol production, the total amount of cortisol (AUC) produced and the values of FIQ after the exercise program were analyzed. A negative correlation between AUC and FIQ values was noticed (Figure 4). These results showed a negative correlationship that high values of cortisol indicate a better quality of life in these patients (lower FIQ values).

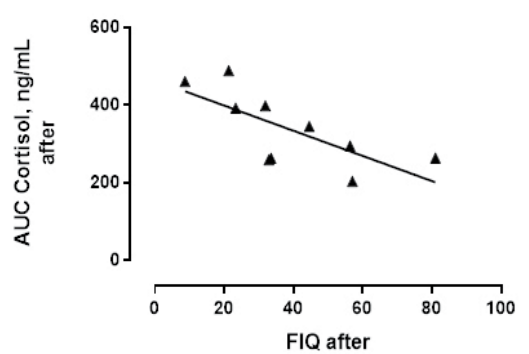

Figure 4) Correlationship of total cortisol daily production and Fibromyalgia Impact Questionnaire (FIQ) scores after the vibratory platform exercise program. Ten volunteers participated in the exercise program over eight weeks. Analysis: Pearson $r=0.7194, p=0.0190$.

\section{DISCUSSION}

Pain and its effects on the individual's life have a subjective character and the quantification of this impact is relevant to evaluate the effectiveness of any intervention. The FIQ is a questionnaire used for this purpose, which is translated and validated for many languages (18).

The initial FIQ values i.e., 58.07 from the volunteers participating in this pilot study are in agreement with those obtained in the studies of a Spanish population i.e., 59. The vibratory platform exercise program promoted an improvement in the quality of life of the volunteers, which could be perceived by the decrease of the FIQ score after participation in the exercise program (19).

The improvement in quality of life in volunteers with fibromyalgia through the practice of physical activity programs is being validated in the literature (19) and the results are in agreement with these data. López-Pousa et al. studied individuals (men and women) diagnosed with fibromyalgia who take a walk for six days. The volunteers presented low scores for the FIQ applied after the exercise period. This shows that there was an improvement in the quality of life of these people. The same results were observed by Sevimli et al. (20), who worked with different types of exercise and the influence on the quality of life of patients with fibromyalgia. Patients who participated in an aerobic exercise program on the ground and another group that did aerobic activities in the pool for three months had a reduction in FIQ scores after the proposed exercise period, without any significant differences between activities (20).

The improvement in the FIQ scores of fibromyalgia patients undergoing exercise is associated with a reduction in pain. The mechanisms related to the improvement of the pain of these patients, after participation in a regular 
exercise program, are not yet fully elucidated, however, there are indications that the performance of regular exercises by patients with fibromyalgia may increase the activity of brain regions related to modulation of pain $(21,22)$.

In situations of physical and/or mental stress, a healthy person's cortisol can increase by up to 20 times (23). Physiologically, any perception of physical alteration or tissue injury is transmitted through the brainstem to the middle eminence, where the adrenocorticotrophic hormone (ACTH) is secreted into the pituitary-portal system. In the anterior pituitary, the stimulus for ACTH secretion occurs, which stimulates the adrenal cortex to release cortisol. However, under pain chronic stress, the hypocortisolism has been reported. This effect is characterized by the decreased availability and/ or effects of cortisol in the body $(23,24)$. On the other hand, under other chronic stressors, there is a decrease in the number of cortisol receptors, as a consequence of the hypersecretion of this hormone for a long time (25).

In fibromyalgia, the chronic pain is the main complaint of patients and causes the state of prolonged stress, where hypocortisolism becomes an expected phenomenon. Despite this, there are few studies evaluating this disease and this condition is not well established in fibromyalgia patients. Riva et al. (11) observed that the cortisol concentrations of patients undergoing physiotherapeutic treatment for fibromyalgia presented lower cortisol values, when compared to the control group, which characterizes the hypocortisolism. However, patients with fibromyalgia that were not performing any type of treatment for disease, presented higher cortisol values (26). In addition, there are studies that show that patients with fibromyalgia did not present any significant difference in cortisol concentrations when compared to women control (27). Data from this pilot study demonstrated that the population of fibromyalgic volunteers presented cortisol concentrations similar to the healthy population in all samples, corroborating with Wingenfeld et al. (27).

The results showed that the volunteers in the control group already had cortisol rhythmicity before the exercise program and that it was maintained after eight weeks of practice. However, after training, these volunteers had higher concentrations of cortisol in the upon awakening samples. These results are in agreement with Batista et al. (1), who studied the effects of a Tantric Yoga program on healthy volunteers. This population had an increase in morning cortisol and a significant decrease in feelings such as irritability, tension, fatigue, fear and anxiety. This work indicates that the Yoga program contributed to the eustress of the studied population. There was no change in the total production of cortisol evaluated by the area under the curve (AUC).

The rhythmicity of the daily cortisol production of the fibromyalgic volunteers who participated in this study was compromised, since there was a significant decrease in the concentrations of this hormone in the nocturnal samples. After exercise, an improvement in rhythmicity was observed, which can be noticed by the significant drop in cortisol at 6:00 p.m. and 10:00 p.m. There were no significant changes in the daily cortisol production in this population. Studies of the effect of physical activity practice under the cortisol rhythmicity in fibromyalgic patients are non-existent. However, in a non-fibromyalgic population, Batista et al. (1) pointed out an improvement in rhythmicity in the population of volunteers who practiced Yoga for seven weeks.

Aerobic exercises have important effects on the relief of the symptoms of patients with fibromyalgia $(28,29)$. In the physical exercise between $30 \%$ and $40 \%$ of $\mathrm{VO}_{2}$ max or when an exercise exceeds the anaerobic threshold of the individual, we observed an increase in ACTH concentrations and, after 10 minutes, elevation of cortisol concentrations (30). Genc et al. (30) found that ACTH concentrations were not altered after a program of aerobic exercise on treadmill for six weeks; in addition, cortisol concentrations were also not reduced after the program. By the way, patients with fibromyalgia who performed a single maximal physical exercise session showed reductions in the concentrations of ACTH and cortisol (31).

Correlating the FIQ scores with the cortisol values and AUC, after the exercise program, it was observed that higher concentrations of cortisol lead to lower FIQ scores. This result suggests that the improvement in the symptoms and quality of life of these patients is related to adjustment of cortisol production, thus contributing to a greater internal balance and, consequently, to eustress, as described by Batista et al. (1).

\section{CONCLUSION}

Based on the results, it is suggested that patients with fibromyalgia, who participated in a vibratory platform treatment program for eight weeks, showed an improvement in the quality of life and this may be related to an improvement in the cortisol rhythm.
This pilot study presents limitations related to the reduced number of patients who participated in the study because of patient adherence throughout protocol.

\section{ACKNOWLEDGEMENT}

The authors wish to thank the volunteers and the support team. This work is a result of the master dissertation of Kelly Regina Francisco supervised by Prof Dr. Hidetake Imasato and defended by the Programa de Pós-Graduação Interunidades em Bioengenharia from the Universidade de São Paulo and was supported by CNPQ. We state there are no competing financial interests in the work described.

\section{DISCLOSURES}

Wan, Tashakkor and Mancini: None

Leipsic: Circle Cardiovascular Imaging, GE, Samsung, Phillips

Raggi: None

\section{REFERENCES}

1. Batista JC, Souza AL, Ferreira HA, et al. Acute and chronic effects of tantric yoga practice on distress index. J Altern Complement Med. 2015;21(11):681-685.

2. Sapolsky RM. Stress hormones: Good and bad. Neurobiol Dis. 2000;7(5):540-542.

3. Dinan TG. Glucocorticoids and the genesis of depressive illness. A psychobiological model. Br J Psychiatry. 1994;164(3):365-771.

4. Selye $H$. The general adaptation syndrome and the diseases of adaptation. J Clin Endocrinol Metab. 1946;6:117-230.

5. Selye H.A Syndrome produced by diverse nocuous agents. Nature 1936 p. 32-.

6. Griffin JF. Stress and immunity: A unifying concept. Vet Immunol Immunopathol. 1989;20(3):263-312.

7. Van de Kar LD, Richardson-Morton KD, Rittenhouse PA. Stress: Neuroendocrine and pharmacological mechanisms. Methods Achiev Exp Pathol. 1991;14:133-173.

8. Franks BD. What is stress? QUEST 1994. p. 1-7.

9. Cavalcante AB, Sauer JF, Chalot SD, et al. The prevalence of fibromyalgia: A literature review. Bras J Reumatol 2006. p. 40-8.

10. Fatima G, Das SK, Mahdi AA, et al. Circadian rhythm of serum cortisol in female patients with fibromyalgia syndrome. Indian J Clin Biochem. 2013;28(2):181-184.

11. Riva R, Mork PJ, Westgaard RH, et al. Fibromyalgia syndrome is associated with hypocortisolism. Int J Behav Med. 2010;17(3):223-233.

12. Isomeri R, Mikkelsson $\mathrm{M}$, Latikka $\mathrm{P}$, et al. Effects of amitriptyline and cardiovascular fitness training on pain patients with primary fibromyalgia. Journal of Musculoskeletal Pain 1993. p. 253-260.

13. Burckhardt CS, Mannerkorpi K, Hedenberg L, et al. A randomized, controlled clinical trial of education and physical training for women with fibromyalgia. J Rheumatol. 1994;21(4):714-720.

14. Martin L, Nutting A, MacIntosh BR, et al. An exercise program in the treatment of fibromyalgia. J Rheumatol. 1996;23(6):1050-1053.

15. Alentorn-Geli E, Padilla J, Moras G, et al. Six weeks of whole-body vibration exercise improves pain and fatigue in women with fibromyalgia. J Altern Complement Med. 2008;14(8):975-981.

16. Marques AP, Santos AMB, Assumpção A, et al. Validation of the Brazilian version of the fibromyalgia impact questionnaire (FIQ) validation of the Brazilian version of the fibromyalgia impact questionnaire (FIQ). Rev Bras Reumatol 2006. p. 24-31.

17. Pires Da Rocha MC, Figueiredo De Martino MM, Grassi-Kassisse DM, et al. Stress among nurses: an examination of salivary cortisol levels on work and day off. Rev Esc Enferm USP. 2013;47(5):1194-1201.

18. Marques AP, Santos AMB, Assumpção A, et al. Validation of the brazilian version of the Fibromyalgia Impact Questionnaire (FIQ). Rev Bras Reumatol 2006. p. 24-31. 
19. López-Pousa S, Bassets Pagès G, Monserrat-Vila S, et al. Sense of well-being in patients with fibromyalgia: Aerobic exercise program in a mature forest - A pilot study. Evid Based Complement Alternat Med. 2015.

20. Sevimli D, Kozanoglu E, Guzel R, et al. The effects of aquatic, isometric strength-stretching and aerobic exercise on physical and psychological parameters of female patients with fibromyalgia syndrome. J Phys Ther Sci. 2015;27(6):1781-1786.

21. Gracely RH, Ambrose KR. Neuroimaging of fibromyalgia. Best Pract Res Clin Rheumatol. 2011;25(2):271-284.

22. Ellingson LD, Stegner AJ, Schwabacher IJ, et al. Exercise strengthens central nervous system modulation of pain in fibromyalgia. Brain Sci. 2016;6(1).

23. Petrelluzzi KF, Garcia MC, Petta CA, et al. Salivary cortisol concentrations, stress and quality of life in women with endometriosis and chronic pelvic pain. Stress. 2008;11(5):390-397.

24. Yehuda R, Teicher MH, Trestman RL, et al. Cortisol regulation in posttraumatic stress disorder and major depression: a chronobiological analysis. Biol Psychiatry. 1996;40(2):79-88.

25. Haas DA, George SR. Single or repeated mild stress increases synthesis and release of hypothalamic corticotropin-releasing factor. Brain Res. $1988 ; 461(2): 230-237$

26. Catley D, Kaell AT, Kirschbaum C, et al. A naturalistic evaluation of cortisol secretion in persons with fibromyalgia and rheumatoid arthritis. Arthritis Care Res. 2000;13(1):51-61.

27. Wingenfeld K, Heim C, Schmidt I, et al. HPA axis reactivity and lymphocyte glucocorticoid sensitivity in fibromyalgia syndrome and chronic pelvic pain. Psychosom Med. 2008;70(1):65-72.

28. Thomas EN, Blotman F. Aerobic exercise in fibromyalgia: A practical review. Rheumatol Int. 2010;30(9):1143-1150.

29. Vural M, Berkol TD, Erdogdu Z, et al. Evaluation of the effectivenes of an aerobic exercise program and the personality characteristics of patients with fibromyalgia syndrome: a pilot study. J Phys Ther Sci. 2014;26(10):1561-1565.

30. Genc A, Tur BS, Aytur YK, et al. Does aerobic exercise affect the hypothalamic-pituitary-adrenal hormonal response in patients with fibromyalgia syndrome? J Phys Ther Sci. 2015;27(7):2225-2231.

31. Torgrimson-Ojerio B, Ross RL, Dieckmann NF et al. Preliminary evidence of a blunted anti-inflammatory response to exhaustive exercise in fibromyalgia. J Neuroimmunol. 2014;277(1-2):160-167. 